\title{
Lemierre-like Syndrome and Infective Endocarditis: A Triumph of Eponyms
}

\author{
Andrea Amabile ${ }^{1}$ and Arnar Geirsson ${ }^{1}$ \\ ${ }^{1}$ Yale University School of Medicine
}

May 31, 2021

Commentary:

Lemierre-like Syndrome and Infective Endocarditis: A Triumph of Eponyms

Andrea Amabile MD, Arnar Geirsson MD

Division of Cardiac Surgery, Department of Surgery, Yale School of Medicine, New Haven, Connecticut

Corresponding Author: Dr. Arnar Geirsson, MD

Professor of Surgery Division of Cardiac Surgery

Yale School of Medicine BB204, 330 Cedar Street P.O. Box 208039, 06510, New Haven, CT

Business phone: 203-785-6214

Fax: 203-785-3346

Email: arnar.geirsson@yale.edu

Conflict of interest: none

Word count: 722

Text

The use of medical eponyms is widespread across hospital wards and medical school halls worldwide, and it clearly indicates that the history of medicine is much more than an arid catalogue of discoveries. It is estimated that more than 8,000 medical eponyms exist ${ }^{1}$, and a certain degree of debate has been recently generated on eponyms being potentially archaic and scientifically misleading despite being of undoubted fascination as they embed different medical traditions and cultures $^{2}$. At the intersection of otolaryngology, infectious disease and vascular surgery, Lemierre Syndrome (LS) was first described by Andre Lemierre in 1936 as 'anaerobic postanginal sepsis', consisting of septic thrombophlebitis of the internal jugular vein secondary to uncontrolled oropharyngeal infection by Fusobacteriumspecies ${ }^{3}$.

In this issue of the Journal, Morishige and colleagues ${ }^{4}$ reported the case of an uncommon variant of this rare syndromic disease, which has an annual incidence of 3.6 cases per million people in its classic presentation ${ }^{5}$. The Authors presented a case which stood out under multiple standpoints:

1. First, the pathophysiology was unique. They presented a case of infective endocarditis secondary to thrombophlebitis in the left vertebral vein which was secondary to pharyngitis caused by methicillinsensitive Staphylococcus Aureus. They identified this condition as a rare LS variant. Interestingly, the primary focus of infection had spread to the left ventricular wall and created a pedunculated, 
encapsulated, purulent abscess attached to the trabeculae carnae of the anterior ventricular wall. Additional eponymic signs of systemic septic embolization were appreciated, namely Osler nodes (painful, red, raised lesions on extremities) and Janeway lesions (non-tender, small haemorrhagic lesions on the palms and soles), but not Roth-Litten spots (small, white-centered retinal hemorrhages) - all of which may be typical in patients with infective endocarditis.

2. Secondly, the decision-making process for optimal treatment was multidisciplinary, as suggested by the guidelines on infective endocarditis ${ }^{6}$. The Authors had cardiologists, infectious diseases physicians and neurologists involved in rapidly assessing the patient pre-operatively and ruling out potential contraindications to early surgery such as embolic stroke ${ }^{7}$. Notably, the patient was neurologically compromised at presentation with acute cerebral and cerebellar dysfunction and a Glasgow Coma Scale of 7 . However, after careful evaluation of the size $(<10 \mathrm{~mm})$ and locations (frontal cortex, caudate nucleus, and left cerebellar hemisphere) of the hyperdense lesions at brain magnetic resonance imaging, the neurologic status was mainly attributed to sepsis-associated encephalopathy rather than septic embolization, thus allowing for prompt surgical control of the infective source.

3. Finally, the outcome was excellent. The patient underwent early surgery (transseptal abscess excision) followed by long-term antimicrobial therapy to control the primary source of infection and anticoagulation therapy to prevent left vertebral vein thrombophlebitis from recurring, with complete normalization of neurologic status at discharge and no relapsing at latest follow-up.

In conclusion, Morishige and colleagues must be congratulated for their comprehensive, collaborative, multidisciplinary efforts which led to the successful treatment of a rare and highly comorbid syndromic condition.

\section{References}

1. Ferguson RP, Thomas D. Medical eponyms. $J$ Community Hosp Intern Med Perspect. 2014;4(3):10.3402/jchimp.v3404.25046.

2. Whitworth JA. Should eponyms be abandoned? No. BMJ.2007;335(7617):425.

3. Lemierre A. ON CERTAIN SEPTICÆMIAS DUE TO ANAEROBIC ORGANISMS.The Lancet. 1936;227(5874):701-703.

4. Morishige S, Oe M, Goto T, Ishimaru T. Successful Perioperative Management of a Case of Infective Endocarditis Secondary to a Lemierre's Syndrome Variant with Severe Neurological Manifestation. J Card Surg. 2021;In press.

5. Hagelskjaer Kristensen L, Prag J. Lemierre's syndrome and other disseminated Fusobacterium necrophorum infections in Denmark: a prospective epidemiological and clinical survey. Eur J Clin Microbiol Infect Dis. 2008;27(9):779-789.

6. Habib G, Lancellotti P, Antunes MJ, et al. 2015 ESC Guidelines for the management of infective endocarditis: The Task Force for the Management of Infective Endocarditis of the European Society of Cardiology (ESC)Endorsed by: European Association for Cardio-Thoracic Surgery (EACTS), the European Association of Nuclear Medicine (EANM).European Heart Journal. 2015;36(44):3075-3128.

7. Yanagawa B, Pettersson GB, Habib G, et al. Surgical Management of Infective Endocarditis Complicated by Embolic Stroke: Practical Recommendations for Clinicians. Circulation.2016;134(17):1280-1292. 